\title{
A single dose of dapagliflozin, an SGLT-2 inhibitor, induces higher glycosuria in GCK- and HNF1A-MODY than in type 2 diabetes mellitus
}

\author{
J. Hohendorff ${ }^{1,2}$ - M. Szopa ${ }^{1,2}$ - J. Skupien ${ }^{1,2}$ - M. Kapusta ${ }^{3}$ B. Zapala ${ }^{3}$ T. Platek ${ }^{3}$. \\ S. Mrozinska ${ }^{1,2} \cdot$ T. Parpan ${ }^{4}$ - W. Glodzik ${ }^{5}$ - A. Ludwig-Galezowska ${ }^{6} \cdot$ B. Kiec-Wilk ${ }^{1,2}$. \\ T. Klupa ${ }^{1,2} \cdot$ M. T. Malecki ${ }^{1,2}$
}

Received: 26 February 2017 / Accepted: 1 June 2017 / Published online: 7 June 2017

(C) The Author(s) 2017. This article is an open access publication

\begin{abstract}
Aims SGLT2 inhibitors are a new class of oral hypoglycemic agents used in type 2 diabetes (T2DM). Their effectiveness in maturity onset diabetes of the young (MODY) is unknown. We aimed to assess the response to a single dose of $10 \mathrm{mg}$ dapagliflozin in patients with Hepatocyte Nuclear Factor 1 Alpha (HNF1A)-MODY, Glucokinase (GCK)-MODY, and type 2 diabetes.

Methods We examined 14 HNF1A-MODY, 19 GCKMODY, and 12 type 2 diabetes patients. All studied individuals received a single morning dose of $10 \mathrm{mg}$ of dapagliflozin added to their current therapy of diabetes. To assess the response to dapagliflozin we analyzed change in urinary glucose to creatinine ratio and serum 1,5-Anhydroglucitol (1,5-AG) level.

Results There were only four patients with positive urine glucose before dapagliflozin administration (one with HNF1A-MODY, two with GCK-MODY, and one with T2DM), whereas after SGLT-2 inhibitor use, glycosuria
\end{abstract}

M. T. Malecki

malecki_malecki@yahoo.com

1 Department of Metabolic Diseases, Jagiellonian University Medical College, Krakow, Poland

2 Department of Metabolic Diseases, University Hospital, Krakow, Poland

3 Department of Clinical Biochemistry, Jagiellonian University Medical College, Krakow, Poland

4 Brothers Hospitallers' of St. John of God Hospital, Krakow, Poland

5 Sanatio Medical Center, Krakow, Poland

6 Center for Medical Genomics OMICRON, Jagiellonian University Medical College, Krakow, Poland occurred in all studied participants. Considerable changes in mean glucose to creatinine ratio after dapagliflozin administration were observed in all three groups $(20.51 \pm 12.08$, $23.19 \pm 8.10$, and $9.84 \pm 6.68 \mathrm{mmol} / \mathrm{mmol}$ for HNF1AMODY, GCK-MODY, and T2DM, respectively, $p<0.001$ for all comparisons). Post-hoc analysis revealed significant differences in mean glucose to creatinine ratio change between type 2 diabetes and each monogenic diabetes in response to dapagliflozin ( $p=0.02, p=0.003$ for HNF1-A and GCK MODY, respectively), but not between the two MODY forms $(p=0.7231)$. Significant change in serum 1,5-AG was noticed only in T2DM and it was $-6.57 \pm$ $7.34 \mathrm{mg} / \mathrm{ml}(p=0.04)$.

Conclusions A single dose of dapagliflozin, an SGLT-2 inhibitor, induces higher glycosuria in GCK- and HNF1AMODY than in T2DM. Whether flozins are a valid therapeutic option in these forms of MODY requires long-term clinical studies.

Keywords HNF1A · GCK · MODY - SGLT2 ·

Dapagliflozin

$\begin{array}{ll}\text { Abbreviations } \\ \text { 1,5-AG } & \text { 1,5-Anhydroglucitol } \\ \text { apo-M } & \text { Apolipoprotein M } \\ \text { BMI } & \text { Body mass index } \\ \text { CKD-EPI } & \begin{array}{l}\text { Chronic kidney disease epidemiology } \\ \text { collaboration }\end{array} \\ \text { eGFR } & \text { Estimated glomerular filtration rate } \\ \text { FPG } & \text { Fasting plasma glucose } \\ \text { GCK } & \text { Glucokinase } \\ \text { GCR } & \text { Urinary glucose to creatinine ratio } \\ \text { HbA1c } & \text { Hemoglobin A1c } \\ \text { HNF1A } & \text { Hepatocyte nuclear factor 1 alpha } \\ \text { hs-CRP } & \text { High-sensitivity C-reactive protein }\end{array}$


MODY Maturity onset diabetes of the young

SGLT2 Sodium/Glucose co-transporter 2

SU Sulfonylurea

T1DM Type 1 diabetes mellitus

T2DM Type 2 diabetes mellitus

\section{Introduction}

Maturity Onset Diabetes of the Young (MODY) is estimated to account for $1-2 \%$ of all cases of diabetes, which may correspond to hundreds of thousands of patients in Europe. The most typical clinical features of MODY include early age of diagnosis (usually in the $2 \mathrm{nd}$ and $3 \mathrm{rd}$ decade of life), positive family history, autosomal dominant mode of inheritance, absence of autoantibodies typical for type 1 diabetes mellitus (T1DM), and lack of obesity [1]. Most MODY cases result from mutations within the hepatocyte nuclear factor 1 alpha $(H N F 1 A)$ and glucokinase $(G C K)$ genes [2, 3].

Mutations in the HNF1A gene, which encodes a transcription factor that regulates the expression of many other genes, lead to insulin secretion impairment [4]. As a result, patients with HNF1A-MODY require pharmacotherapy and often develop chronic complications [5, 6]. These patients typically respond well to treatment with sulfonylurea, which increases insulin release from beta cells and is currently considered the treatment of choice for patients with HNF1A-MODY [7, 8]. As the nature of the beta-cell defect in HNF1A-MODY is progressive, this treatment frequently requires further intensification with additional hypoglycemic agents. The extra-pancreatic features of HNF1AMODY include glycosuria due to a low renal threshold for glucose [9], which has been linked to decreased sodium/ glucose co-transporter 2 (SGLT2) expression in tubular cells [10].

Meanwhile, in GCK-MODY glucose homeostasis is less affected than in HNF1A-MODY. GCK-MODY subjects are characterized by mild fasting hyperglycemia. It is generally accepted that most diabetic GCK mutation carriers do not need hypoglycemic treatment [11]. They usually do not develop advanced chronic microvascular complications of diabetes, although, the prevalence of background retinopathy is rather high, reaching 30\% according to British data [12]. Moreover, there is also some evidence on abnormalities in surrogate cardiovascular outcomes in $G C K$ mutation carriers [13]. Therefore, clinicians would probably consider initiation of treatment in some GCK-MODY patients. Regrettably, pharmacological treatment including either insulin, or oral hypoglycemic agents, such as metformin or $\mathrm{SU}$, turned out to be ineffective in this form of MODY, probably due to the fact that the switch-on of the counterregulatory response occurs at higher glucose level than in healthy subjects $[14,15]$. So, there is still a need to test new available treatments in both of the two most frequent subtypes of MODY [1]. This is particularly important because thanks to the widening use of next-generation sequencing a growing number of patients with a genetic diagnosis of MODY will be identified [16, 17].

Recently a new group of oral hypoglycemic agentsSGLT2 inhibitors-were introduced into the market and made available for patients with diabetes. These inhibitors block the low-affinity, high capacity glucose transporter located in the proximal tubule in the kidneys that is responsible for $90 \%$ of glucose reabsorption. Therefore, SGLT2 inhibitors can reduce plasma glucose levels by producing glycosuria [18]. A single dose of the SGLT2 inhibitor dapagliflozin was shown to be enough to produce glycosuria and the effect lasted up to $24 \mathrm{~h}[19,20]$. As mentioned above, the expression of SGLT2 and the function of the SGLT2 protein are reduced among HNF1A-MODY individuals [10]. Therefore, it can hypothesized that response to SGLT2 inhibitors, such as dapagliflozin, may be compromised among HNF1A-MODY individuals, thus influencing their efficacy in these patients.

\section{Aim of the study}

We aimed to assess the response to a single dose of $10 \mathrm{mg}$ dapagliflozin in patients with HNF1A-MODY and to compare it with T2DM and GCK-MODY individuals by measuring changes in urinary glucose to creatinine ratio (GCR) and in serum 1,5-anhydroglucitol (1,5-AG) level, which indirectly reflects episodes of glycosuria. 1,5-AG is a short-term marker of glycemic control (mainly postprandial) that corresponds to the preceding 1-2 weeks. In a state of glycosuria, renal reabsorption of 1,5-AG is decreased, as this particle competes with glucose for reabsorption in the proximal renal tubule, which results in its lower serum level [21-24]. Additionally. we assess GCR change as a diagnostic tool to distinguish between T2DM and MODY.

\section{Materials and methods}

\section{Study population}

The studied group included 14 HNF1A-MODY patients, 19 GCK-MODY individuals, and 12 T2DM subjects. All MODY patients had a heterozygous loss-of-function mutation either in the HNFIA or GCK gene identified by direct DNA sequencing. HNF1A-MODY patients were members of 13 families, whereas GCK-MODY individuals 
were members of 17 families. T2DM individuals were ascertained as described earlier [25].

We included patients with at least 2 years duration of diabetes and without diabetic kidney disease defined as Chronic Kidney Disease Epidemiology Collaboration (CKD-EPI) estimated glomerular filtration rate (eGFR) $<60$ $\mathrm{ml} / \mathrm{min} / 1.73 \mathrm{~m}^{2}$ diagnosed prior to inclusion in the study. We excluded patients with a hemoglobin A1c (HbA1c) $>9.0 \%(75 \mathrm{mmol} / \mathrm{mol})$, those who were on insulin or steroid therapy, or taking drugs affecting renal function (e.g., loop diuretics). Pregnancy was also an exclusion criterion. We gathered clinical data on the study individuals from their medical records. Additionally, we collected the information from the standard questionnaire and performed a basic physical examination. All studied individuals received a single morning dose of $10 \mathrm{mg}$ of dapagliflozin. The patients' current therapy, including use of other hypoglycemic agents, was not changed. To assess the glycosuric response to dapagliflozin the following analyses were performed in all subjects: (1) three measurements of urinary glucose and creatinine concentrations: one in the morning on the dapagliflozin administration day (Day 0), one 8-12 h after administration of the drug, and one in the morning on Day +1 ; (2) two measurements of serum 1,5-AG level: one in the morning on Day 0 and one in the morning on Day +1 . Additionally, we performed two measurements of fasting plasma glucose (FPG): one in the morning on Day 0 , and one in the morning on $D a y+1$, as well as two daily glucose profiles performed with glucose meters by participants (fasting, before and $2 \mathrm{~h}$ after main meals): one on the day before drug administration (Day -1) and one on the dapagliflozin administration day. Urine glucose concentration below $1.0 \mathrm{mmol} / \mathrm{l}$ was considered negative. Urinary GCR was calculated for all three urine measurements. 1,5AG was measured in fasting conditions using a competitive inhibition enzyme immunoassay (ELISA; Cloud Clone Corp., Houston, TX 77,082, USA). The assay has a sensitivity of $0.7 \mu \mathrm{g} / \mathrm{ml}$, the intra- and inter-assay coefficients of variation of the ELISA were $<10 \%$. The absorbance was finally measured in a microplate reader (BioTek Instruments, Winooski, VT, USA). The study was approved by the local Bioethics Committee. All participants gave written informed consent.

\section{Statistical analysis}

Statistical analysis was performed using Statistica Software v. 12.0 (StatSoft, Tulsa, OK, USA) and MedCalc Statistical Software v 16.8.4 (MedCalc Software bvba, Ostund, Belgium). All performed tests were two-tailed and a $p$-value $<0.05$ was considered significant. The Kolmogorov-Smirnov test was used to determine the normal distribution of variables. $p$-values were calculated with the $t$-test and the one-way ANOVA or nonparametric Kruskall-Wallis test, followed by post hoc-tests. Multivariate linear regression analysis (backward selection) was used to assess differences in mean GCR and 1,5-AG change between studied groups after adjustment for gender, age, diabetes duration, body mass index (BMI), eGFR, FPG, and HbA1c. Diagnostic performance (i.e., the ability of GCR after dapagliflozin administration to identify MODY and T2DM) was assessed using the receiver operating characteristics (ROC) curve. The standard error (SE) of the area under the ROC curve (AUC) and 95\% confidence intervals (95\% CI) were calculated using DeLong's method [26].

\section{Results}

The characteristics of the study participants are presented in Table 1. There were differences between the study groups for age, BMI and HbA1c. The patients with T2DM were older, more obese, and were characterized by worse glycemic control than HNF1A-MODY and GCK-MODY subjects what is in line with the way how groups were defined. In post-hoc analyses, we found no differences between HNF1A-MODY and GCK-MODY subjects according to age, BMI and HbAlc. There were no differences between study groups in terms of sex distribution and kidney function. During the study examination we diagnosed one patient with chronic kidney disease (eGFR $47 \mathrm{ml} /$ $\min / 1.73 \mathrm{~m}^{2}$ ) and his results were included in the analyses. Results presented by that patient were not detected as outliers. All study participants were free from proliferative diabetic retinopathy. Almost all GCK gene mutation carriers were on dietary therapy only; only one GCK-MODY patient (a 67-year old female) was on metformin. Among the 14 HNF1A-MODY individuals, 12 were on SU, one patient (a 30-year female with polycystic ovary syndrome) was on metformin, and one was on dietary therapy only. Slightly more than half of patients with T2DM were on combined therapy with metformin and SU or DPP4 inhibitors; other T2DM patients were on metformin monotherapy.

There were only four patients with positive urine glucose before dapagliflozin administration-one patient with HNF1A-MODY, two subjects with GCK-MODY, and one individual with T2DM. After $8-12 \mathrm{~h}$ of dapagliflozin administration glycosuria occurred in all studied patients and the effect persisted for $24 \mathrm{~h}$ in every participant. Significant differences in urinary GCR after $24 \mathrm{~h}$ of dapagliflozin administration were observed in the studied groups as this rose by $20.51 \pm 12.08,23.19 \pm 8.10$, and $9.84 \pm 6.68$ $\mathrm{mmol} / \mathrm{mmol}$ for HNF1A-MODY $(p=0.0000)$, GCKMODY $(p=0.0000)$, and T2DM $(p=0.0003)$, respectively. These mean changes in urinary GCR were different 
Table 1 Clinical characteristic and biochemical measurements of the study group

\begin{tabular}{|c|c|c|c|c|}
\hline Characteristic & HNF1A-MODY & GCK-MODY & $\mathrm{T} 2 \mathrm{DM}$ & $p$-value \\
\hline$N$ & 14 & 19 & 12 & NA \\
\hline Male/female $(n)$ & $7 / 7$ & $7 / 12$ & $8 / 4$ & $0.17^{\mathrm{a}}$ \\
\hline Age at examination (years) & $34.1 \pm 11.0$ & $40.3 \pm 10.8$ & $61.8 \pm 5.6$ & $0.0000^{\mathrm{b}}$ \\
\hline Time from diabetes diagnosis (years) & $11.4 \pm 6.8$ & $8.7 \pm 7.1$ & $5.8 \pm 4.2$ & $0.10^{\mathrm{b}}$ \\
\hline $\operatorname{BMI}\left(\mathrm{kg} / \mathrm{m}^{2}\right)$ & $24.4 \pm 4.9$ & $23.4 \pm 2.8$ & $31.3 \pm 5.3$ & $0.0000^{\mathrm{b}}$ \\
\hline eGFR CKD-EPI (ml/min/1.73 m²) & $98.1 \pm 15.1$ & $99.9 \pm 15.6$ & $87.7 \pm 15.9$ & $0.10^{\mathrm{b}}$ \\
\hline $\mathrm{HbA1c}(\%, \mathrm{mmol} / \mathrm{mol})$ & $6.0 \pm 0.7,42.0 \pm 5.3$ & $6.4 \pm 0.4,46.0 \pm 2.0$ & $6.9 \pm 0.9,52.0 \pm 7.5$ & $0.03^{\mathrm{c}}$ \\
\hline hsCRP (mg/l) & $0.52 \pm 0.30$ & $1.12 \pm 1.57$ & $1.60 \pm 1.15$ & $0.09^{\mathrm{b}}$ \\
\hline $\begin{array}{l}\text { GCR (Day } 0) \text {-patients with positive urinary glucose } \\
\text { only }(\mathrm{mmol} / \mathrm{mmol}, n)\end{array}$ & $0.15,1$ & $0.17 \pm 0.18,2$ & $2.01,1$ & NA \\
\hline $\begin{array}{l}\text { GCR change (8-12 } \mathrm{h} \text { after dapagliflozin administration) } \\
(\mathrm{mmol} / \mathrm{mmol})\end{array}$ & $35.64 \pm 14.10$ & $38.72 \pm 9.65$ & $19.37 \pm 18.76$ & $0.001^{\mathrm{b}}$ \\
\hline GCR change $($ Day $+1-$ Day 0$)(\mathrm{mmol} / \mathrm{mmol})$ & $20.51 \pm 12.08$ & $23.19 \pm 8.10$ & $9.84 \pm 6.68$ & $0.001^{\mathrm{b}}$ \\
\hline 1,5-AG (Day 0) (mg/ml) & $8.75 \pm 8.43$ & $19.61 \pm 9.22$ & $26.72 \pm 9.03$ & $0.0000^{\mathrm{b}}$ \\
\hline 1,5-AG change $($ Day $+1-$ Day 0$)(\mathrm{mg} / \mathrm{ml})$ & $-0.09 \pm 4.21$ & $-0.86 \pm 4.16$ & $-6.57 \pm 7.34$ & $0.02^{\mathrm{b}}$ \\
\hline FPG (Day 0) (mmol/l) & $5.60 \pm 1.02$ & $6.82 \pm 0.71$ & $7.73 \pm 2.25$ & $0.005^{\mathrm{c}}$ \\
\hline FPG change (Day +1-Day 0$)(\mathrm{mmol} / \mathrm{l})$ & $-0.14 \pm 0.90$ & $-0.50 \pm 0.72$ & $-0.23 \pm 1.20$ & $0.51^{\mathrm{b}}$ \\
\hline Average daily glucose in SMBG $($ Day -1$)(\mathrm{mmol} / \mathrm{l})$ & $6.02 \pm 1.03$ & $6.99 \pm 0.50$ & $7.60 \pm 1.77$ & $0.008^{\mathrm{c}}$ \\
\hline Average daily glucose in SMBG (Day 0) (mmol/l) & $5.81 \pm 0.87$ & $6.90 \pm 0.59$ & $7.32 \pm 1.58$ & $0.002^{\mathrm{c}}$ \\
\hline $\begin{array}{l}\text { Average daily glucose change in SMBG (Day 0-Day } \\
-1)(\mathrm{mmol} / \mathrm{l})\end{array}$ & $-0.21 \pm 0.61$ & $-0.09 \pm 0.51$ & $-0.27 \pm 1.27$ & $0.84^{\mathrm{c}}$ \\
\hline
\end{tabular}

Data are presented as mean \pm standard deviation

1,5-AG 1,5-Anhydroglucitol, FPG fasting plasma glucose, $G C R$ glucose to creatinine ratio, $h s C R P$ high sensitive C-reactive protein, $N A$ not applicable, $n$ number of cases, $S M B G$ self-monitoring of blood glucose

$\chi^{2}$ test

${ }^{\mathrm{b}}$ One-way analysis of variance (ANOVA)

${ }^{\mathrm{c}}$ Kruskal-Wallis test

between the groups $(p=0.001)$. Post-hoc analysis revealed significant differences in mean GCR rise between the T2DM and MODY forms of diabetes $(p=0.02$ and $p=$ 0.003 for HNF1-MODY and GCK-MODY, respectively); however, no difference between the HNF1A-MODY and GCK-MODY groups were observed $(p=0.72)$. Nevertheless, in multivariate linear regression $(\mathrm{R} 2=27 \%)$, the only significant variable remained BMI $(p=0.0001)$, but not type of diabetes. Similar results in GCR change in the evening on Day 0 (after 8-12 h of dapagliflozin administration) were observed.

As expected, mean 1,5-AG level on Day 0 was the lowest in HNF1A-MODY. The significant change in mean serum 1,5-AG was noticed only in T2DM, for which it was $-6.57 \pm 7.34 \mathrm{mg} / \mathrm{ml}(p=0.04)$, but not in HNF1A-MODY $(-0.10 \pm 4.21 \mathrm{mg} / \mathrm{ml}, p=0.94)$ or GCK-MODY $(-0.86 \pm$ $4.16 \mathrm{mg} / \mathrm{ml}, p=0.38)$. In the one-way ANOVA, a considerable difference in mean 1,5-AG change among the studied groups was found $(p=0.02)$. Post-hoc analyses revealed significant discrepancy in 1,5-AG change between T2DM and HNF1A-MODY $(p=0.04)$ and a borderline difference between T2DM and GCK-MODY $(p=0.07)$. In the multivariate linear regression $(\mathrm{R} 2=27 \%)$, the only significant variable was initial serum 1,5-AG level $(p=$ 0.0009).

Mean initial FPG was significantly lower in the HNF1MODY group than in the GCK-MODY and T2DM groups. There were no significant differences in FPG between the GCK-MODY and T2DM groups. Subgroup analyses revealed significant mean FPG reduction in GCKMODY $(0.50 \pm 0.72 \mathrm{mmol} / \mathrm{l}, \quad p=0.007)$, but not in HNF1A-MODY $(0.14 \pm 0.90 \mathrm{mmol} / \mathrm{l}, p=0.56)$ and T2DM $(0.23 \pm 1.20 \mathrm{mmol} / \mathrm{l}, p=0.52)$. ANOVA analysis revealed no differences in the degree of FPG reduction at this sample size.

Mean initial average daily glucose level based on selfmonitoring of blood glucose (SMBG) was the lowest in the HNF1A-MODY $(p=0.01)$. No significant reduction in mean average daily glucose was found in the studied groups $(-0.21 \pm 0.61 \mathrm{mmol} / \mathrm{l}, p=0.2154 ;-0.09 \pm 0.51 \mathrm{mmol} / 1, p$ $=0.4686 ;-0.27 \pm 1.27 \mathrm{mmol} / \mathrm{l}, p=0.4710$ for $\mathrm{HNF} 1 \mathrm{~A}-$ MODY, GCK-MODY and T2DM, respectively). 
Fig. 1 Receiver operating characteristics (ROC) curves showing the discriminative accuracy of glucose to creatine ratio (GCR) change after dapagliflozin administration to distinguish between the diabetes subgroups: a.T2DM and HNF1A-MODY; b T2DM and GCK-MODY; and $\mathbf{c}$ HNF1AMODY and GCK-MODY
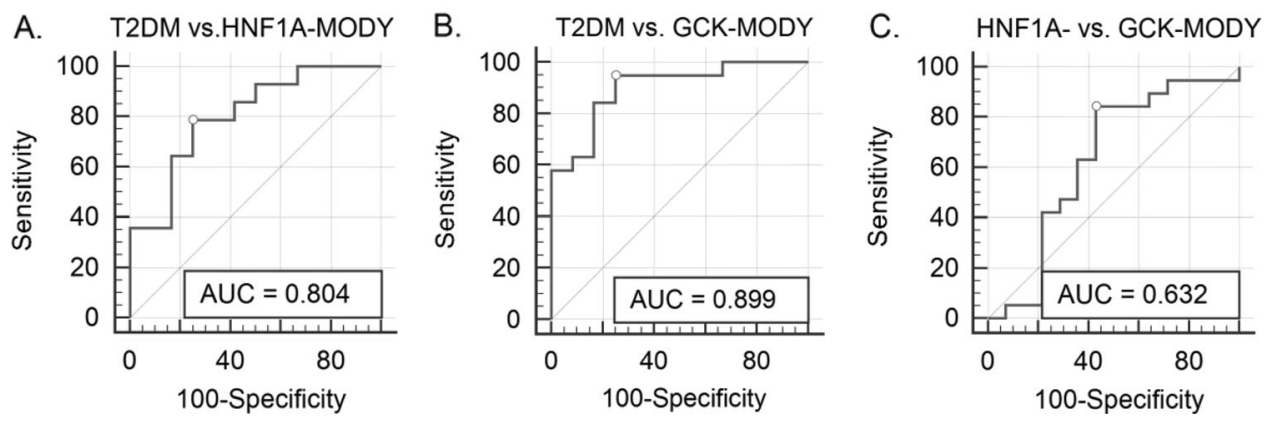

Additionally, we assessed change in GCR (after $24 \mathrm{~h}$ of dapagliflozin administration) as a biomarker in differential diagnosis for subtypes of diabetes. The ROC curves showing the ability of GCR change to differentiate between the three subtypes of diabetes are presented in Fig. 1. The ROC curve analysis performed for T2DM and HNF1AMODY revealed an AUC of 0.80 (95\% CI 0.60-0.93) with sensitivity $78.6 \%$ (95\% CI 49.2-95.3) and specificity $75.0 \%$ (95\% CI 42.8-94.5). The results for T2DM and GCKMODY were: 0.90 (0.74-0.98), 94.7\% (74.0-99.9) and 75.0\% (42.8-94.5), respectively. For GCK-MODY and HNF1A-MODY, AUC was 0.63 (95\% CI 0.45-0.79), with a sensitivity of $84.2 \%$ (95\% CI 60.4-96.6\%) and specificity of $57.1 \%$ (95\% CI $28.9-82.3 \%$ ).

\section{Discussion}

In this small-scale study, we assessed the response to a single morning dose of $10 \mathrm{mg}$ of dapagliflozin in HNF1AMODY patients and compared to T2DM and GCK-MODY subjects. SGLT2 inhibition was previously shown to be clinically effective, safe and reduce cardiovascular outcomes and mortality as well as death from any cause in T2DM patients. Desirable effects of SGLT2 inhibitors include no risk of hypoglycemia, body weight loss and a small reduction of systolic blood pressure. The most common side effects are genitourinary infections [27-30]. The SGLT2 inhibitor dapagliflozin also induces glycosuria in healthy volunteers $[19,31]$. While there was one case report on a patient undergoing combined therapy with SU and dapagliflozin in ABCC8-MODY [32], to the best of our knowledge, no published studies have investigated the effect of SGLT2 inhibitors in patients with GCK-MODY or HNF1A-MODY. Here, in this short, $24 \mathrm{~h}$ clinical experiment, we show for the first time that dapagliflozin induces glycosuria in both HNF1A-MODY and GCK-MODY patients and reduces FPG in GCK-MODY.

Our finding concerning dapagliflozin-induced glycosuria in HNF1A mutation carriers is especially interesting. Glycosuria in HNF1A-MODY results from reduced expression of the SGLT2, which is under the transcriptional control of
HNF1A. In HNF1A-deficient animals, there is $80-90 \%$ reduction in SGLT2 expression, which correlates with a reduction in SGLT2 activity [10]. Furthermore, glycosuria that occurs in HNF1A-deficient animals cannot be compensated by the activity of other glucose transporters such as SGLT1 (sodium/glucose co-transporter 1) and SGLT3 (sodium/glucose co-transporter 3) [10]. SGLT2 is responsible for $90 \%$ of glucose reabsorption. However, in humans, SGLT2 inhibitors block only $30-50 \%$ of the glucose load, and a number of explanations have been proposed to explain this phenomenon $[33,34]$. One potential cause of this effect is that SGLT1 works at submaximal capacity under physiological conditions, with the residual capacity of SGLT1 revealed upon SGLT2 inhibition [35]. However, the precise mechanisms of incomplete inhibition of glucose load with SGLT2 inhibitors and the interplay between SGLT1 and SGLT2 transporters are not fully understood. In our study, we observed no difference between HNF1A and $G C K$ mutation carriers in the effects of dapagliflozin administration. This may mean that the degree of SGLT2 inhibition with dapagliflozin does not depend on baseline activity of the SGLT2 protein, which is lower in HNF1AMODY patients. Surprisingly, the GCR change was greater in both the HNF1A-MODY and GCK-MODY group than in the T2DM group in our study. However, one must take into consideration the significant clinical differences observed among T2DM patients and those with MODY in our study. Indeed, when we subsequently performed a multivariate linear regression analysis, BMI was the only significant predictive variable for GCR. Due to obvious differences in the way the study groups were defined, we were unable to match our T2DM and MODY patients according to age and BMI.

Serum 1,5-AG level is a short-term marker of glycemic control that competes with glucose for reabsorption [36]. It was shown that 1,5-AG level is reduced in HNF1A-MODY; however, its discriminative accuracy is not high enough for it to be widely used in clinical practice [24, 37]. In our study mean serum 1,5-AG level change after dapagliflozin administration was statistically significant only in T2DM; this finding is in contrast with change in glycosuria, which was lowest in that group. Multivariate linear regression 
showed that 1,5-AG level change depends on initial 1,5-AG level-which was higher in T2DM than in both MODY forms. A lower change in MODY could also results from more stable glycemia over a period of a day. Moreover, while 1,5-AG level is an independent marker of glycemic control and glycosuria over 7-10 days, in our 24-h study the full impact of dapagliflozin on the level of this marker could not be seen due to the short-time nature of the experiment.

A proper diagnosis of MODY is crucial in order to conduct adequate treatment. While genetic testing becomes more and more available, there is still a need for cheap, easy biochemical markers. Some of them were reported in earlier research (apo-M, hs-CRP, 1,5-anhydroglucitol, ghrelin) [24, 38-40], nevertheless, none of them have been introduced to the clinical practice. Drug response-based diagnostic tests are quite common in medicine, especially in endocrinology, for example, dexamethasone suppression tests, the clonidine test, or the metoclopramide test. While our drug-based test results are promising, one should be cautious with expectations of using the test in routine practice.

Furthermore, we did not evaluate the long-term clinical efficacy of dapagliflozin in MODY patients in the present study. However, we did prove that dapagliflozin works in MODY patients as well as in those with T2DM. Therefore, SGLT2 inhibitors should be considered as an additional therapeutic option in MODY patients in future. A safety aspect that will have to be examined is a possible rise in ketogenesis in these individuals, a phenomenon that was earlier reported in T2DM and T1DM [41]. This seems to be particularly important in HNFA1-MODY, as a few cases of diabetic ketoacidosis were reported in this monogenic diabetes [42, 43].

Interestingly, we observed a significant drop in FPG in GCK-MODY patients after one tablet of dapagliflozin. It is a well known phenomenon that all pharmacological treatment used so far in this form MODY was ineffective due to counterregulatory mechanisms $[11,14,15]$. Most GCKMODY patients do not need any hypoglycemic treatment. However, clinicians would probably consider treatment initiation in some GCK-MODY individuals, especially in patients with relatively common nonproliferative retinopathy, those with $\mathrm{HbA} 1 \mathrm{c}>7.0 \%(53.0 \mathrm{mmol} / \mathrm{mol})$ and $/$ or "overlapping" type 2 diabetes. Thus, identification of an effective and safe drug for these subjects would be of clinical value. It can be hypothesized that SGLT-2 inhibitors could effectively lower blood glucose level in these patients due to their specific mode of action.

\section{Conclusion}

To summarize, we have shown that a single dose of dapagliflozin, an SGLT-2 inhibitor, induces higher glycosuria in
HNF1A- and GCK-MODY than in T2DM. There was no difference in response to dapagliflozin between $H N F 1 A$ and GCK mutation carriers, despite a previously reported reduction in SGLT2 expression among HNF1A-MODY individuals. Whether flozins, such as dapagliflozin, are a valid therapeutic option as an adjunctive therapy in these MODY patients requires long-term clinical studies.

Acknowledgements The study was supported by the European Foundation for the Study of Diabetes (EFSD) New Horizons grant for Prof. T. Klupa and a Diabetes Poland research grant, 2014, for Dr. M. Szopa for the project titled: Evaluation of the effectiveness of biomarkers in screening for HNF1A-MODY.

\section{Compliance with ethical standards}

Conflict of interest The authors declare that they have no competing interests.

Ethical approval All procedures performed in studies involving human participants were in accordance with the ethical standards of the institutional and/or national research committee and with the 1964 Helsinki declaration and its later amendments or comparable ethical standards.

Open Access This article is distributed under the terms of the Creative Commons Attribution 4.0 International License (http://creativecommons.org/licenses/by/4.0/), which permits unrestricted use, distribution, and reproduction in any medium, provided you give appropriate credit to the original author(s) and the source, provide a link to the Creative Commons license, and indicate if changes were made.

\section{References}

1. B.M. Shields, S. Hicks, M.H. Shepherd, K. Colclough, A.T. Hattersley, S. Ellard, Maturity-onset diabetes of the young (MODY): how many cases are we missing? Diabetologia 53(12), 2504-2508 (2010)

2. A. Stride, A.T. Hattersley, Different genes, different diabetes: lessons from maturity-onset diabetes of the young. Ann. Med. 34 (3), 207-216 (2002)

3. J. Kropff, M.P. Selwood, M.I. McCarthy, A.J. Farmer, K.R. Owen, Prevalence of monogenic diabetes in young adults: a community-based, cross-sectional study in oxfordshire, UK. Diabetologia 54(5), 1261-1263 (2011)

4. K. Yamagata, Roles of HNF1 $\alpha$ and $\mathrm{HNF} 4 \alpha$ in pancreatic $\beta$-cells: lessons from a monogenic form of diabetes (MODY). Vitam. Horm. 95, 407-423 (2014)

5. M. Szopa, J. Wolkow, B. Matejko, J. Skupien, T. Klupa, I. Wybranska, I. Trznadel-Morawska, B. Kiec-Wilk, M. Borowiec, M.T. Malecki, Prevalence of retinopathy in adult patients with GCK-MODY and HNF1A-MODY. Exp. Clin. Endocrinol. Diabetes 123(9), 524-528 (2015)

6. B. Isomaa, M. Henricsson, M. Lehto, C. Forsblom, S. Karanko, L. Sarelin, M. Häggblom, L. Groop, Chronic diabetic complications in patients with MODY3 diabetes. Diabetologia 41(4), 467-473 (1998) 
7. E.R. Pearson, B.J. Starkey, R.J. Powell, F.M. Gribble, P.M. Clark, A.T. Hattersley, Genetic cause of hyperglycaemia and response to treatment in diabetes. Lancet 362(9392), 1275-1281 (2003)

8. M. Shepherd, B. Shields, S. Ellard, O. Rubio-Cabezas, A.T. Hattersley, A genetic diagnosis of HNF1A diabetes alters treatment and improves glycaemic control in the majority of insulintreated patients. Diabet. Med. 26(4), 437-441 (2009)

9. T.J. McDonald, S. Ellard, Maturity onset diabetes of the young: identification and diagnosis. Ann. Clin. Biochem. 50(5), 403-415 (2013)

10. M. Pontoglio, D. Prié, C. Cheret, A. Doyen, C. Leroy, P. Froguel, G. Velho, M. Yaniv, G. Friedlander, HNF1alpha controls renal glucose reabsorption in mouse and man. EMBO. Rep. 1(4), 359-365 (2000)

11. A.J. Chakera, A.M. Steele, A.L. Gloyn, M.H. Shepherd, B. Shields, S. Ellard, A.T. Hattersley, Recognition and management of individuals with hyperglycemia because of a heterozygous glucokinase mutation. Diabetes Care 38(7), 1383-1392 (2015)

12. A.M. Steele, B.M. Shields, K.J. Wensley, K. Colclough, S. Ellard, A.T. Hattersley, Prevalence of vascular complications among patients with glucokinase mutations and prolonged, mild hyperglycemia. JAMA 311(3), 279-286 (2014)

13. M. Szopa, G. Osmenda, G. Wilk, B. Matejko, J. Skupien, B. Zapala, W. Mlynarski, T. Guzik, M.T. Malecki, Intima-media thickness and endothelial dysfunction in GCK and HNF1AMODY patients. Eur. J. Endocrinol. 172(3), 277-283 (2015)

14. A. Stride, B. Shields, O. Gill-Carey, A.J. Chakera, K. Colclough, S. Ellard, A.T. Hattersley, Cross-sectional and longitudinal studies suggest pharmacological treatment used in patients with glucokinase mutations does not alter glycaemia. Diabetologia 57(1), 54-56 (2014)

15. E. Guenat, G. Seematter, J. Philippe, E. Temler, E. Jequier, L. Tappy, Counterregulatory responses to hypoglycemia in patients with glucokinase gene mutations. Diabetes Metab. 26(5), 377-384 (2000)

16. M. Szopa, A. Ludwig-Galezowska, P. Radkowski, J. Skupien, B. Zapala, T. Platek, T. Klupa, B. Kiec-Wilk, M. Borowiec, W. Mlynarski, P. Wolkow, M.T. Malecki, Genetic testing for monogenic diabetes using targeted next-generation sequencing in patients with maturity-onset diabetes of the young. Pol. Arch. Med. Wewn. 125(11), 845-851 (2015)

17. S. Ellard, H. Lango Allen, E. De Franco, S.E. Flanagan, G. Hysenaj, K. Colclough, J.A. Houghton, M. Shepherd, A.T. Hattersley, M.N. Weedon, R. Caswell, Improved genetic testing for monogenic diabetes using targeted next-generation sequencing. Diabetologia 56(9), 1958-1963 (2013)

18. M.A. Abdul-Ghani, L. Norton, R.A. DeFronzo, Efficacy and safety of SGLT2 inhibitors in the treatment of type 2 diabetes mellitus. Curr. Diab. Rep. 12(3), 230-238 (2012)

19. B. Komoroski, N. Vachharajani, D. Boulton, D. Kornhauser, M. Geraldes, L. Li, M. Pfister, Dapagliflozin, a novel SGLT2 inhibitor, induces dose-dependent glucosuria in healthy subjects. Clin. Pharmacol. Ther. 85(5), 520-526 (2009)

20. B. Komoroski, N. Vachharajani, Y. Feng, L. Li, D. Kornhauser, M. Pfister, Dapagliflozin, a novel, selective SGLT2 inhibitor, improved glycemic control over 2 weeks in patients with type 2 diabetes mellitus. Clin. Pharmacol. Ther. 85(5), 513-519 (2009)

21. J.B. McGill, T.G. Cole, W. Nowatzke, S. Houghton, E.B. Ammirati, T. Gautille, M.J. Sarno, Circulating 1,5-anhydroglucitol levels in adult patients with diabetes reflect longitudinal changes of glycemia: a U.S. trial of the GlycoMark assay. Diabetes Care 27(8), 1859-1865 (2004)

22. K.M. Dungan, J.B. Buse, J. Largay, M.M. Kelly, E.A. Button, S. Kato, S. Wittlin, 1,5-anhydroglucitol and postprandial hyperglycemia as measured by continuous glucose monitoring system in moderately controlled patients with diabetes. Diabetes Care 29(6), 1214-1219 (2006)

23. E.S. Kilpatrick, B.G. Keevilt, K.L. Richmond, P. Newland, G.M. Addison, Plasma 1,5-anhydroglucitol concentrations are influenced by variations in the renal threshold for glucose. Diabet. Med. 16(6), 496-499 (1999)

24 J. Skupien, S. Gorczynska-Kosiorz, T. Klupa, K. Wanic, E.A. Button, J. Sieradzki, M.T. Malecki, Clinical application of 1,5anhydroglucitol measurements in patients with hepatocyte nuclear factor-1alpha maturity-onset diabetes of the young. Diabetes Care 31(8), 1496-1501 (2008)

25. M. Szopa, M. Kapusta, B. Matejko, T. Klupa, T. Koblik, B. KiecWilk, M. Borowiec, M.T. Malecki, Comparison of glomerular filtration rate estimation from serum creatinine and cystatin $\mathrm{C}$ in HNF1A-MODY and other types of diabetes. J. Diabetes Res. (2015). doi:10.1155/2015/183094

26. E.R. DeLong, D.M. DeLong, D.L. Clarke-Pearson, Comparing the areas under two or more correlated receiver operating characteristic curves: a nonparametric approach. Biometrics 44(3), 837-845 (1988)

27. 2016 Guidelines on the management of diabetic patients, A position of diabetes Poland. Clin. Diabet. 5(Suppl. A), A16-A18 (2016)

28. S.E. Inzucchi, R.M. Bergenstal, J.B. Buse, M. Diamant, E. Ferrannini, M. Nauck, A.L. Peters, A. Tsapas, R. Wender, D.R. Matthew, Management of hyperglycaemia in type 2 diabetes, 2015: a patient-centred approach. Update to a Position Statement of the American Diabetes Association and the European Association for the Study of DiabetesDiabetologia 58, 429-442 (2015).

29. B. Zinman, C. Wanner, J.M. Lachin, D. Fitchett, E. Bluhmki, S. Hantel, M. Mattheus, T. Devins, O.E. Johansen, H.J. Woerle, U.C. Broedl, S.E. Inzucchi, EMPA-REG OUTCOME investigators: Empagliflozin, cardiovascular outcomes, and mortality in type 2 diabetes. N. Engl. J. Med. 373(22), 2117-2128 (2015)

30. R.P. Monica Reddy, S.E. Inzucchi, SGLT2 inhibitors in the management of type 2 diabetes. Endocrine 53(2), 364-372 (2016)

31. R.A. DeFronzo, M. Hompesch, S. Kasichayanula, X. Liu, Y. Hong, M. Pfister, L.A. Morrow, B.R. Leslie, D.W. Boulton, A. Ching, F.P. LaCreta, S.C. Griffen, Characterization of renal glucose reabsorption in response to dapagliflozin in healthy subjects and subjects with type 2 diabetes. Diabetes. Care 36(10), 3169-3176 (2013)

32. A.K. Ovsyannikova, O.D. Rymar, E.V. Shakhtshneider, V.V. Klimontov, E.A. Koroleva, N.E. Myakina, M.I. Voevoda, ABCC8-related maturity-onset diabetes of the young (MODY12): clinical features and treatment perspective. Diabetes Ther. 7(3), 591-600 (2016)

33. J.J. Liu, T. Lee, R.A. DeFronzo, Why do SGLT2 inhibitors inhibit only $30-50 \%$ of renal glucose reabsorption in humans? Diabetes 61(9), 2199-2204 (2012)

34. M.A. Abdul-Ghani, R.A. DeFronzo, L. Norton, Novel hypothesis to explain why SGLT2 inhibitors inhibit only $30-50 \%$ of filtered glucose load in humans. Diabetes 62(10), 3324-3328 (2013)

35. V. Vallon, S.C. Thomson, Targeting renal glucose reabsorption to treat hyperglycaemia: the pleiotropic effects of SGLT2 inhibition. Diabetologia 60(2), 215-225 (2017)

36. J.B. Buse, J.L. Freeman, S.V. Edelman, L. Jovanovic, J.B. McGill, Serum 1,5-anhydroglucitol (GlycoMark): a short-term glycemic marker. Diabetes. Technol. Ther. 5(3), 355-363 (2003)

37. A. Pal, A.J. Farmer, C. Dudley, M.P. Selwood, B.A. Barrow, R. Klyne, J.P. Grew, M.I. McCarthy, A.L. Gloyn, K.R. Owen, Evaluation of serum 1,5 anhydroglucitol levels as a clinical test to differentiate subtypes of diabetes. Diabetes Care 33(2), 252-257 (2010) 
38. S.A. Mughal, R. Park, N. Nowak, A.L. Gloyn, F. Karpe, H. Matile, M.T. Malecki, M.I. McCarthy, M. Stoffel, K.R. Owen, Apolipoprotein M can discriminate HNF1A-MODY from type 1 diabetes. Diabet. Med. 30(2), 246-250 (2013)

39. G. Thanabalasingham, N. Shah, M. Vaxillaire, T. Hansen, T. Tuomi et al.. A large multi-centreeuropean study validates highsensitivity C-reactive protein (hsCRP) as a clinical biomarker for the diagnosis of diabetes subtypes. Diabetologia 54(11), 2801-2810 (2011)

40. N. Nowak, J. Hohendorff, I. Solecka, M. Szopa, J. Skupien, B. Kiec-Wilk, W. Mlynarski, M.T. Malecki, Circulating ghrelin level is higher in HNF1A-MODY and GCK-MODY than in polygenic forms of diabetes mellitus. Endocrine 50(3), 643-649 (2015)
41. H. Qiu, A. Novikov, V. Vallon, Ketosis and diabetic ketoacidosis in response to SGLT2 inhibitors: basic mechanisms and therapeutic perspectives. Diabetes Metab. Res. Rev. (2017). doi:10. 1002/dmrr.2886 [Epub ahead of print]

42. S. Pruhova, P. Dusatkova, D. Neumann, E. Hollay, O. Cinek, J. Lebl, Z. Sumnik, Two cases of diabetic ketoacidosis in HNF1AMODY linked to severe dehydration: is it time to change the diagnostic criteria for MODY? Diabetes Care 36(9), 2573-2574 (2013)

43. Egan A.M., Cunningham A., Jafar-Mohammadi B., Dunne F.P., Diabetic ketoacidosis in the setting of HNF1A-maturity onset diabetes of the young. BMJ Case Reports. (2015). doi:10.1136/ bcr-2014-209163 